\title{
Kinect-Based Rehabilitation Training Assistant System Research And Implementation
}

\author{
Huaqiang Jiang \\ ${ }^{1}$ College of Mechatronics Engineering and Automation, \\ Shanghai University, Shanghai, China \\ ${ }^{2}$ College of Info Science and Engineering of Hangzhou \\ Normal University, China \\ e-mail: jhq@hznu.edu.cn
}

\author{
Xiang Jie \\ ${ }^{2}$ College of Info Science and Engineering of Hangzhou \\ Normal University, China \\ e-mail: xia_ngj_ie@hotmail.co
}

\begin{abstract}
A Kinect-based rehabilitation training assistant system was devised aims to improve the posture accuracy in limbrecovery rehabilitation for stroke patient with hemiplegia. Kinect features in capturing user's limb node data, and together with rule of weighted Euclidean distance, this system compares obtained data with the stored standard ones, and then to output the data about the accuracy of rehabilitation postures. It is easy to install, simple to use and low in cost, etc. which may well-assist stroke patients with hemiplegia rehabilitation training.
\end{abstract}

Keywords- Kinect; rehabilitation training; DTW algorithm

\section{INTRODUCTION}

Though stroke has become a high incidence disease, with the development of emergency medical treatment, the survival rate has been greatly increased. However, rate of disability due to stroke is rather high; according to relevant materials, the disability rate is as high as $75 \%$ [1]. Usually rehabilitation training postures is not standard for lacking of professional guidance in rehabilitation training, furthermore, part of the central nervous cannot create new network through training and cannot form a new functional cell group network; consequently, it's hard to recover to the fit state. So, a set of good and cheap, and practical rehabilitation training assistant system will play a vital important role in posture correction for stroke patient with hemiplegia.

Currently, rehabilitation training for stroke patient with hemiplegia is mainly guided by people. Only a few equipment manufacturers have orthoses, limb motion rehabilitation and other auxiliary training equipment to assist the rehabilitation training. Researches on rehabilitation training system are rare, either. It mainly concentrated in few engineering universities, and research content focuses mainly on the development of rehabilitation training devices. Tsinghua University's research is comparatively deep, such as the development of lower-limb rehabilitation training vehicle, neurological disability rehabilitation training mechanism, and upper-limb multiplemotion rehabilitation training robot, etc. [2]

Kinect is a 3D somatosensory camera, which can input spontaneous data like human gestures, image and voice, etc. Users don't have to wear any sensors and interaction with computer can be achieved through making motions in front of Kinect. Kinect helps to get rid of the space limits and patients can manipulate with bare hands. Kinect-based rehabilitation training assistant system has no limits in the common rehabilitation equipment, and a more natural human-computer interaction can be achieved through video, moreover, Kinect's low-in-price makes its popularization possible.

\section{OVERAll Structure Design OF THE System}

Kinect-based rehabilitation training assistant system is used for the physical rehabilitation of stroke patient with hemiplegia. It integrates the application of the theories and techniques in the field of computer graphics, kinematics, mapping and rehabilitation medicine, etc. Moreover, it focuses on posture correction and rehabilitation training assisted with XBOX device, and collects data with Kinect. The system interface shown in Fig. 1.

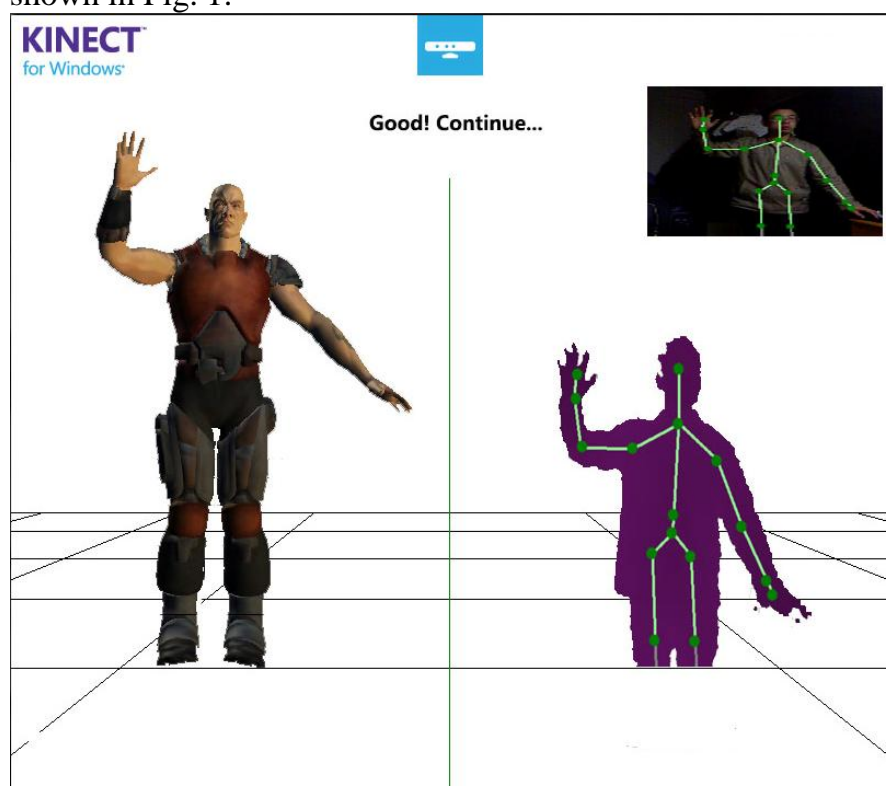

Figure 1. Rehabilitation training preface of Kinect-based rehabilitation training assistant system

The rehabilitation training assistant system mainly consists of three sub-modules: standard posture generation module, rehabilitation posture correction module, feedback and evaluation module. Figure 2 shows the internal relationships between each module.

Correction module: 


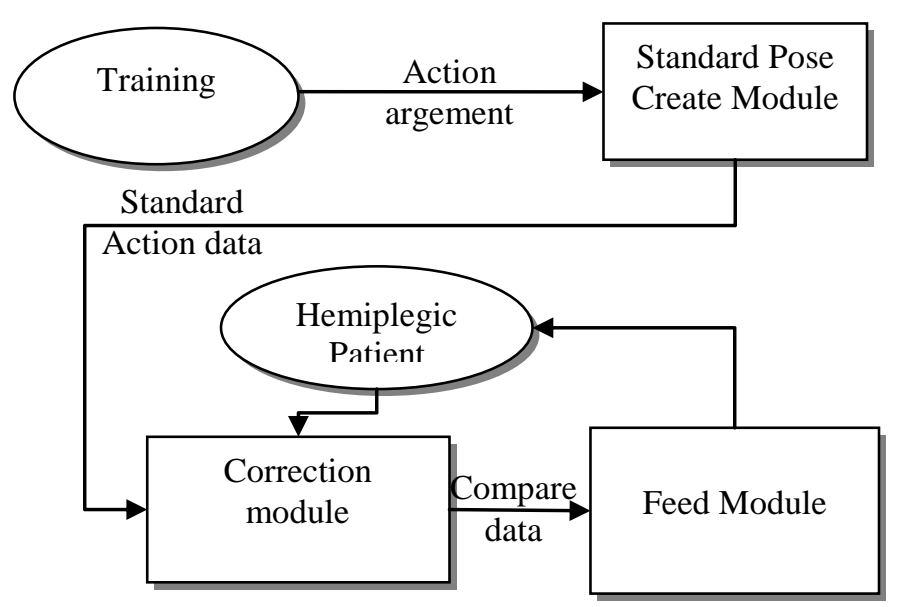

Figure 2. Relationships between each module

(1) Standard posture generation module record the standard action which are devised by rehabilitation training experts in accordance with stroke patients with hemiplegia. Through collecting bone node in experts' action, the strandard data for rehabilitation training was generated.

(2) Rehabilitation posture correction module assists patients with auxiliary training and posture guidance. Obtaining instant data of patients' bone node, and then comparing with the generated standard posture in the first module so as to give guidance.

In order to have a better and vivid understanding of users' traning effect, the correction module will provide five degree rating system for each action. Through rating and fitting each bone node to make users' well-informed of its accuracy.

(3) Feedback and evaluation module is to analyze and evaluate all the collected users' action, and the results will be provided directly to the patients or doctors which can be used as a reference for further treatment.

\section{STANDARD TRAINING ACTION RECORDING AND STORAGE}

Kinect-based rehabilitation training assistant system provides a standard posture generation module which makes the standard training action recording and storage possible. Standard training action is the basis for rehabilitation training. Patients can start rehabilitation training according to the standard action as wll as guided by professional workers, so to personalize rehabilitation training and to improve training effect.

\section{A. Database for Standard Posture}

Database for standard posture stores basic data of standard training. Basic data includes skeleton model of users and color image. Kinect skeleton model consists of 20 bone nodes (Figure 3)[4]. Each node has X, Y, Z three corrdinates. Default video image data is $640 * 480$, RGB32 format. Moreover, database also stores the interval time for current bone node with the startting point of video recording, which is used for synchronization position of bone node and color image.

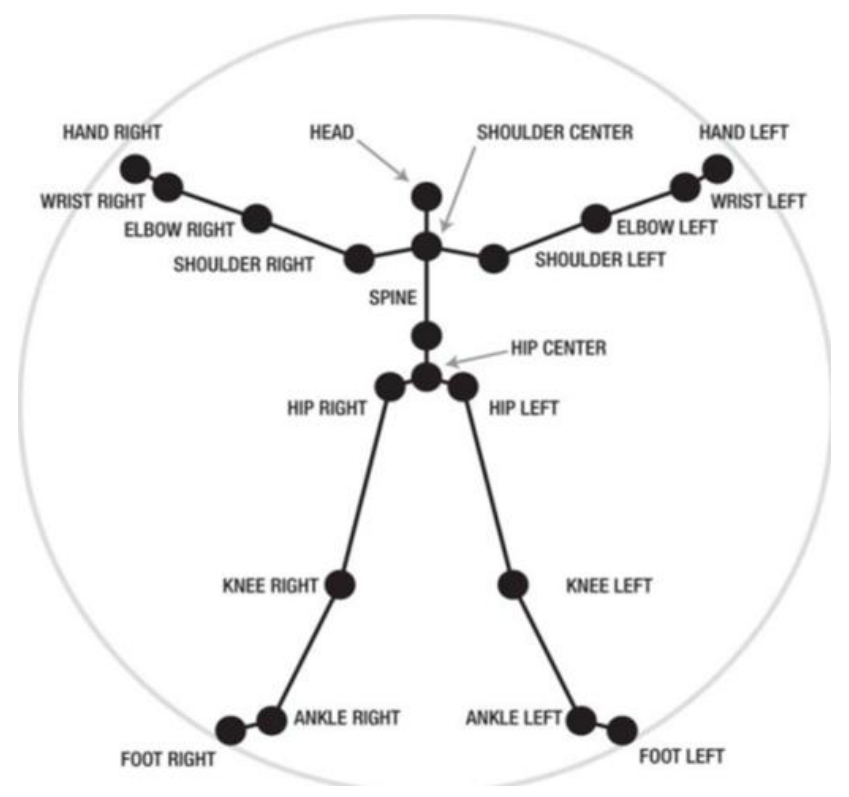

Figure 3. Kinect users' Skeleton model

\section{B. Standard Rehabilitation Training Action Recording Procedure}

Standard rehabilitation action recording procedure is as follows:

(1) select standard posture generation module to check current users' state and wait for strandard posture recording.

(2) click "Start" button by moving arms, create color image data clips and start to recording actions.

(3) record current user's skeleton model, and append color image data to the files.

(4) check interval time between arms and legs movement (if there is no changing for long time) and estimate whether the action clip is complete or not.

(5) if arms and legs motions are continous, then go to step (3) to continue recording, or create ID for a new video recording clip, then go to step (3) to continue recording.

(6) check if arms touch "Stop Recording" button in the course of recording, if yes, then don't save; if it lasts long, then stop recording.

\section{REALIZATION OF REHABILITATION TRAINING ACTION CORRECTION FUNTIONS}

Rehabilitation training action correction funtion is an auxiliary assesment funtion achieved together by action correction module and feedback and judgement module. Its core and difficulty lie in coparison and analysis of the training action. Kinect-based rehabilitation training system mainly uses DTW (Dynamic Time Warping) algorithm [5] to make dynamic comparison and analysis of body position. Through normalization of skeleton data to compare and evaluatet different skeleton models. 


\section{A. Action Comparative Algorithm}

DTW algorithm is a dynamic programming algorithm put forward by H. Sakoe and S. Chiba in 1978, which was initially used for speech recognition. DTW algorithm makes similarity comparison between two sequences (time-correlated) possible. The length of the two sequences may not be the same, while the interval period for sampling the two sequences must be the same.

We define feature space as $\mathrm{F}$, standard training action sequence $X(x 1, x 2, \ldots, x n)$, length $\mathrm{N}$; patients' rehablilitation training action sequence $Y(y 1, y 2, \ldots, y m)$, length $\mathrm{M}$. In order to compare a set of feature $\mathrm{x}$ and $\mathrm{y}$, defining an overhead function c, i.e. the distance between two sequences[6].

Generally, the more similar $\mathrm{x}$ and $\mathrm{y}$ is, the smaller value $\mathrm{c}(\mathrm{x}, \mathrm{y})$ is. Contrarily, the shorter distance the two feature vectors are, the less similar $\mathrm{x}$ and $\mathrm{y}$ is, the greater the value $\mathrm{c}(\mathrm{x}$, $y)$ is. The more distant the vectors are, the greater overheaded it is. Get each c for each feature vector $(x n, y n)$ (when c with $n \in$ [1: N], $m \in[1: M])$, an overhead matrix $\mathrm{C}$ can be obtained, of which $C(n, m)=c(x n, y m))$. Similarity comparison between the two sequences can be transmitted into finding the shortest path between $\mathrm{X}$ and $\mathrm{Y}$ in overhead matrix $\mathrm{C}$.

According to the theory of the shortest path, the optimalized path $\mathrm{P}^{*}$ between $X$ and $Y$ is the shortest path among all possible ones. Then the DTW distance between $X$ and $Y$ is:

$\operatorname{DTW}(X, Y)=c p^{*}(X, Y)=\min \{c p(X, Y) \mid p$ is a $(N, M)$ regular path \}

The system using $\operatorname{DTW}(X, Y)$ to get its value, and then converted into five-degree rating data, and finally back to the screen to suggest the posture accuracy.

\section{B. Human Skeleton Data Normalization}

Since the stored standard action is not consistent with patients', moreover, the distance between body and Kinect device may also lead to various skeleton data, even for the exact same person. In order to fit itself to DTW algorithm, normalization of human skeleton information was needed.

Taking the shoulder center node of the skeleton model as reference, the distance from left shoulder to the right one is standard since it is relatively fixed, so to define skeleton data normalization formula as [7]:

$$
\vec{P}_{\text {norm }}=\frac{\vec{P}-\vec{C}}{|\vec{L}-\vec{R}|}
$$

Among them, $\vec{P}$ is the vector before normalized, $\vec{C}$ is the vector of shoulder center corresponding to the world coordinate system before normalized. $\vec{P}_{\text {norm }}$ is the vector after normalized. $\vec{L}$ and $\vec{R}$ are vectors for left shoulder and right shoulder respectively. So, the normalization of the skeleton data can be achieved.

\section{CONCLUSION}

Kinect-based rehabilitation assistant system provides stroke patients with hemiplegia a cheap and easy-to-operate way for rehabilitation training. Comparison between personal skeleton data and standard action data helps to improve the posture accuracy and decrease the disability rate for stroke patient with hemiplegia. Different from other program, kinect-based rehabilitation training assistant system allows users to operate through body changes and users can be well-informed of its rehabilitation effect instantly. Accuracy of rehabilitation training action is displayed in form of rating, which makes the training process more challenging and interesting, so to get rid of boredom in the training process. This system with low cost and high availability can not only improve rehabilitation effect from the perspective of physical training, but bring patients much fun for life.

On the other hand, since Kinect body senor stores only 20 bone nodes data, and finger node data is missing, which limits the finger training. Therefore, further research for Kinect skeleton model is needed for collecting more exact data and improving its utility, so to popularize the application of the system to more rehabilitation fields.

\section{ACKNOWLEDGMENT}

This research was supported by Zhejiang Education Department Project "Rehabilitation training system based on gesture recognition research" (ID: Y201328957).

\section{REFERENCES}

[1] Shao Wei, Research of virtual reality training system for robot-assisted upper limb rehabilitation [D]. Shanghai Jiao Tong University, 2012.

[2] Science and Technology Department of Tsinghua University, Neurological rehabilitation robot technology and its industrialization for hemiplegia patients.

[3] Microsoft, Kinect for Windows. Learning resources.

[4] [Microsoft, Kinect for Windows. Human-Machine interface guidelines, 2011.

[5] Li Zheng-Xin, Zhang Feng-Ming, Li Ke-Wu, DTW Based Pattern Matching Method for Multivariate Time Series [J]. Pattern Recognition and Artificial Intelligence, 2011(3): 1652-1655.

[6] Qian He-qing, An augmented reality system for education based on gesture recognition using kinect [D]. Shanghai Jiao Tong University, 2011.

[7] Deng Rui, Zhou Ling-ling, Ying Ren-dong. Gesture extraction and recognition research based on Kinect depth data [J]. Application research of computers, 2013. 